\title{
Parametric decay instability near the upper hybrid resonance and anomalous mm-wave scattering in tokamak and stellarator plasmas
}

\author{
$\underline{\text { S.K. Hansen }}{ }^{1,2}$, S.K. Nielsen ${ }^{2}$, M. Salewski ${ }^{2}$, M. Stejner ${ }^{2}$, J. Stober ${ }^{1}$, \\ and the ASDEX Upgrade team ${ }^{1}$ \\ ${ }^{1}$ Max-Planck-Institut für Plasmaphysik, 85748 Garching bei München, Germany, Soeren.Kjer.Hansen@ipp.mpg.de \\ ${ }^{2}$ Department of Physics, Technical University of Denmark, 2800 Kgs. Lyngby, Denmark
}

\section{Introduction and Background}

The parametric decay instability (PDI) is results from a three-wave interaction in which an incident (pump) wave decays into two daughter waves. PDIs may occur in certain media under the influence of radiation with a sufficiently large amplitude, e.g. in tokamak and stellarator plasmas heated or diagnosed by mm-waves from a gyrotron. Energy and momentum conservation in the threewave process impose the selection rules that the sum of the daughter wave frequencies, $\omega$, and wave vectors, $\mathbf{k}$, should equal those of the pump wave,

$$
\omega_{0}=\omega_{1}+\omega_{2}, \quad \mathbf{k}_{0}=\mathbf{k}_{1}+\mathbf{k}_{2} \text {, }
$$

where subscripts 0,1 and 2 refer to the pump wave, and the low- and high-frequency daughter waves, respectively; up-shifted high-frequency daughter waves, satisfying $\omega_{3}=\omega_{0}+\omega_{1}, \mathbf{k}_{3}=\mathbf{k}_{0}+\mathbf{k}_{1}$, also exist due to scattering of the pump wave by the low-frequency daughter waves. Thus, PDIs result in frequency-shifted radiation which interferes with mm-wave plasma diagnostics such as electron cyclotron emission (ECE) or collective Thomson scattering (CTS). In this work we focus on parametric decay of an electromagnetic pump wave into two electrostatic daughter waves, particularly an X-mode pump wave decaying into a high-frequency warm upper hybrid (UH) wave and a low-frequency warm lower hybrid (LH) daughter wave near the upper hybrid resonance (UHR) of the pump wave. The main motivation is observations of strong PDI-like anomalous sidebands during CTS experiments at the ASDEX Upgrade tokamak [1], shown in Fig. 1, and the LHD stellarator [2, 3]. Furthermore, similar observations have been made during 1st harmonic electron cyclotron resonance heating (ECRH) experiments at the Versator II, FT-1 and TCA tokamaks [4-6], as well as at the W VII-A stellarator [7, 8].

\section{Results and Conclusions}

For the PDI in which an electromagnetic X-mode pump wave decays into a warm $\mathrm{UH}$ and a warm $\mathrm{LH}$ wave near the UHR of the pump wave, we have generalized the results of $[4,9,10]$; the details are given in [11]. The dispersion relations describring the warm $\mathrm{LH}$ and warm $\mathrm{UH}$ waves in a simple plasma are given by $[10,11]$, for vanishing $\mathbf{k}_{\|}$(with respect to the equilibrium magnetic field B) they become,

$$
\begin{aligned}
& \omega_{1}^{2}=\omega_{\mathrm{LH}}^{2}\left(1+A_{1} b_{e 1}\right) \\
& \omega_{2}^{2}=\omega_{\mathrm{UH}}^{2}-\omega_{\mathrm{pe}}^{2} A_{2} b_{e 2}
\end{aligned}
$$

where $\omega_{\mathrm{UH}}{ }^{2}=\omega_{\mathrm{pe}}{ }^{2}+\omega_{\mathrm{ce}}{ }^{2}$ is the square of the UH frequency, $\omega_{\mathrm{LH}}{ }^{2}=\omega_{\mathrm{ce}}{ }^{2} \omega_{\mathrm{pi}}{ }^{2} / \omega_{\mathrm{UH}}{ }^{2}$ is the square of the LH frequency, $b_{e 1,2}=m_{e} T_{e} k_{1,2}{ }^{2} /\left(e^{2} B^{2}\right)$ is the square of the wave number normalised by the inverse square of the thermal electron
Larmor radius, $A_{1}=3\left\{\omega_{\mathrm{pe}}{ }^{2} /\left(4 \omega_{\mathrm{UH}}{ }^{2}\right)+\left[T_{i} /\left(Z_{i} T_{e}\right)\right] \omega_{\mathrm{UH}}{ }^{2} / \omega_{\mathrm{pe}}{ }^{2}\right\}$ and $A_{2}=1 /\left[1-\omega_{\mathrm{pe}}{ }^{2} /\left(3 \omega_{\mathrm{ce}}{ }^{2}\right)\right]\left(Z_{i}\right.$ is the ion charge number and $T_{e, i}$ are given in energy units). From Eq. (3) and $k_{2}>$ $0, \omega_{2}<\omega_{\mathrm{UH}}$ for $\omega_{\mathrm{pe}}{ }^{2}<3 \omega_{\mathrm{ce}}{ }^{2}$ and $\omega_{2}>\omega_{\mathrm{UH}}$ for $\omega_{\mathrm{pe}}{ }^{2}>3 \omega_{\mathrm{ce}}{ }^{2}$, indicating that propagating warm $\mathrm{UH}$ waves only exist on the same side of the UHR as the propagating X-mode, for which $\omega_{2}<\omega_{0}<\omega_{\mathrm{UH}}$, when $\omega_{\mathrm{pe}}{ }^{2}<3 \omega_{\mathrm{ce}}{ }^{2}$, generally limiting the occurrence of this kind of PDI to underdense and weakly overdense plasmas, e.g. found in tokamaks and some stellarator experiments. We additionally note that, from Eqs. (1) and (2), $\omega_{1}>\omega_{\mathrm{LH}}, \omega_{2}=\omega_{0}-\omega_{1}<\omega_{0}-\omega_{\mathrm{LH}}$ and $\omega_{3}=\omega_{0}+\omega_{1}>\omega_{0}+\omega_{\mathrm{LH}}$, consistent with the locations of the peaks observed in Fig. 1. In the dipole limit, $\mathbf{k}_{0} \approx \mathbf{0}$ or $b_{e 1} \approx b_{e 2}$, it is possible to derive analytical electric field amplitude thresholds for the above PDI, as done in $[9,10,11]$.

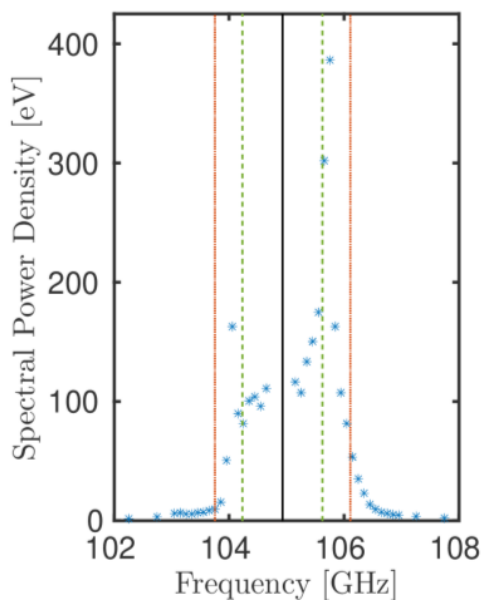

Fig. 1. CTS spectral power density recorded at ASDEX Upgrade in $\mathrm{eV}$. Anomalous peaks shifted in frequency relative to the gyrotron radiation (full line) are observed; the frequency shifts slightly exceed the LH frequency at the UHR (dashed lines), but fall short of the frequency shifts predicted by dipole PDI theory (dotted lines)

The above theory may be used to explain the anomalous sidebands observed during CTS experiments at the ASDEX Upgrade tokamak [1, 10, 11], seen in Fig. 1. In these experiments, depicted in Fig. 2, $105 \mathrm{GHz}$ radiation from a gyrotron is launched from the low-field side of the torus in O-mode and reflected by the high-field side vessel wall, after which part of the reflected radiation may re-enter the plasma in X-mode and ultimately reach the UHR where the PDI may occur. Ray tracing indicates the correlation between the anomalous sidebands in the CTS spectrum and a non-negligible amount of gyrotron radiation reaching the UHR in X-mode implied by the above mechanism [11]. We estimate the electric field 
amplitude of the $\mathrm{X}$-mode radiation using simple ray and beam tracing techniques away from the UHR which are coupled to a $1 \mathrm{D}$ full-wave model close to the UHR [10, 11]. This allows us to convert the analytical pump electric field amplitude thresholds to a CTS gyrotron power threshold necessary to excite the PDI in ASDEX Upgrade. For the experiment under consideration, the CTS gyrotron power threshold is found to be on the order of 100 $\mathrm{kW}$ close to the UHR of the pump wave which is similar to the gyrotron power of $200 \mathrm{~kW}$ used in the experiment, indicating that the anomalous sidebands may be explained by the PDI. We note that the frequency shift predicted by the dipole theory overestimates the experimental one observed in Fig. 1 which may be understood if the warm UH wave is assumed to be forward scattered by the pump wave, following a line of inquiry similar to that of [12].

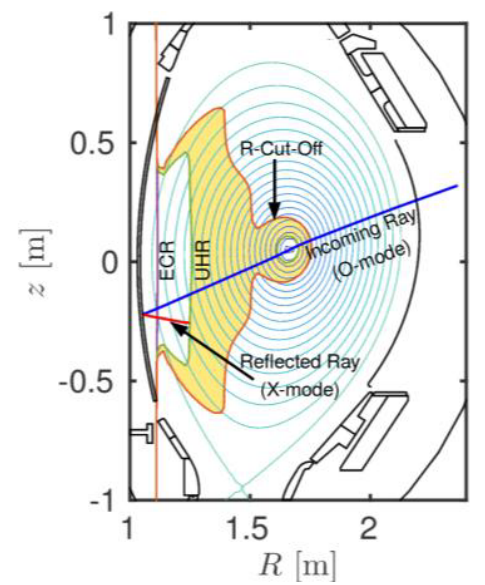

Fig. 2. CTS geometry in ASDEX Upgrade. $105 \mathrm{GHz}$ $\mathrm{O}$-mode gyrotron radiation is injected from the low-field side and reflected off the high-field side wall, after which part of the radiation may re-enter the plasma in X-mode and reach the UHR, where a PDI may occur due to strong field enhancement, provided that absorption at the ECR is not excessisive. X-mode radiation cannot reach the UHR if launched from the low-field side since no propagating $\mathrm{X}$-mode exists in the shaded area

Acknowledgements. This work was supported by a research grant (15483) from VILLUM FONDEN.

\section{References}

1. Nielsen, S.K., Michelsen, P.K., Hansen, S.K., Kors-holm, S.B., Leipold, F., Rasmussen, J., Salewski, M., Schubert, M., Stejner, M., Stober, J., Wagner, D., The ASDEX Upgrade Team Recent development of collective Thomson scattering for magnetically confined fusion plasmas // Phys. Scr. 2017. V. 92. 024001 .
2. Nishiura, M., Kubo, S., Tanaka, K., Seki, R., Ogasawara, S., Shimozuma, T., Okada, K., Kobayashi, S., Mutoh, T., Kawahata, K., Watari, T., LHD Experiment Group, Saito, T., Tatematsu, Y., Korsholm, S. B., Salewski, M. Spectrum response and analysis of $77 \mathrm{GHz}$ band collective Thomson scattering diagnostic for bulk and fast ions in LHD plasmas // Nucl. Fusion. 2014. V. 54. 023006.

3. Kubo, S., Nishiura, M., Tanaka, K., Moseev, D., Ogasawara, S., Shimozuma, T., Yoshimura, Y., Igami, H., Takahashi, H., Tsujimura, T. I., Makino, M., LHD Experiment Group Scattering volume in the collective Thomson scattering measurement using high power gyrotron in the LHD // JINST. 2016. V. 11. C06005.

4. McDermott, F.S., Bekefi, G., Hackett, K.E., Levine, J.S., Porkolab, M. Observation of the parametric decay instability during electron cyclotron resonance heating on the Versator II tokamak // Phys. Fluids. 1982. V. 25, No. 9. P. $1488-1490$.

5. Bulyginsky, D.G., Gusev, V.K., Djachenko, V.V., Irzak, M.A., Kantor, M.Yu., Larionov, M.M., Levin, L.S., Serebreny, G.A., Shustova, N.V. ECR-heating of plasma in FT-1 tokamak and its influence on the ion component // Proc. 11th European Conf. Controlled Fusion and Plasma Physics (Aachen, 1984). Mulhouse: European Physical Society. 1984. V. 1. P. 457-460.

6. Pietrzyk, Z.A., Pochelon, A., Behn, R., Bondeson, A., Dutch, M., Goodman, T.P., Tran, M.Q., Whaley, D.R. Electron cyclotron resonance heating on the TCA tokamak // Nucl. Fusion. 1993. V. 33, No. 2. P. 197-209.

7. Wilhelm, R., Erckmann, V., Janzen, G., Kasparek, W., Müller, G., Raüchle, E., Schüller, P. G., Schwörer, Thumm, M., $W$ VII-A Team Electron cyclotron resonance heating and confinement in the W VII-A stellarator // Plasma Phys. Control. Fusion. 1984. V. 26, No. 12A. P. 1433-1444.

8. Erckmann, V., Janzen, G., Kasparek, W., Müller, G., Räuchle, E., Schüller, P. G., Schwörer, K., Thumm, M., Wilhelm, R., WVII-A Team Wave absorption and transport studies on ECR-heated stellarator plasmas in W VII-A // Proc. 10th Int. Conf. Plasma Physics and Controlled Nuclear Fusion Research (London, 1984). Vienna: International Atomic Energy Agency. 1985. V. 2. P. 419-428.

9. Porkolab, M. Parametric decay instability in ECR heated plasmas // Proc. 2nd Workshop Hot Electron Ring Physics (San Diego, 1981), edited by N. A. Uckan. Alexandria: National Technical Information Service. 1982. V. 1. P. 237-246.

10. Hansen, S.K. Parametric decay instability near the upper hybrid resonance and anomalous scattering from tokamak plasmas // M.Sc. Thesis. Kgs. Lyngby: Technical University of Denmark. 2016.

11. Hansen, S.K., Nielsen, S.K., Salewski, M., Stejner, M., Stober, J., the ASDEX Upgrade team Parametric decay instability near the upper hybrid resonance in magnetically confined fusion plasmas // Submitted to: Plasma Phys. Control. Fusion.

12. Dodin, I.Y. and Arefiev, A.V. Parametric decay of plasma waves near the upper-hybrid resonance // Phys. Plasmas. 2017. V. 24. 032119. 